\title{
ESTUDIO SOBRE LOS CAMBIOS GENERADOS POR LA APLICACIÓN DE INTERNET EN LA FUNCION DE MARKETING EN LAS GRANDES EMPRESAS DE LA CIUDAD DE MANIZALES DURANTE EL PERIODO DE 1997 A 2001
}

Lina María Echeverri Cañas

Palabras claves: Marketing, Internet, Web Site

"Ni la red ni el dispositivo empleado son determinantes para la organización de la compañía: hay que aprender a focalizarse en el usuario." (Doménech, 2001)

RESUMEN EJECUTIVO

En Colombia, la utilización de Internet como herramienta de mercadeo se encuentra en una fase introductoria según el informe final de Conectividad Nacional.(1) De acuerdo con el informe, varias empresas han dirigido sus esfuerzos hacia Internet con el propósito de que éste se constituya como uno de sus principales puntos de venta, transformando la visión tradicional de negocio.

En el grupo de las grandes empresas, una meta clara en el corto plazo es orientar el mercadeo de sus productos directamente hacia el

1 BUSTAMANTE, Claudia Ximena y FAJARDO, Isabel Cristina. Infraestructura de Internet en Colombia: i $n f o r m e$ fin a I deconectividad nacional. Junio de 2001. En: http://www.crt.gov.co/pro yectos/infra_internet_20 00.htm
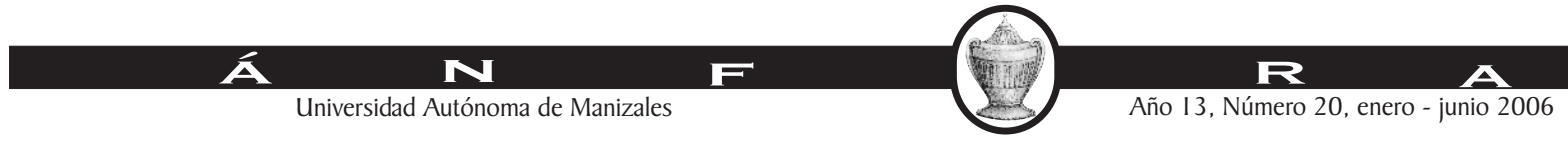
cliente, como soporte fundamental en su modelo de negocio: hacer al consumidor el centro de todas las acciones de la empresa. Por esta razón, se realizó un estudio de los cambios generados por la aplicación de Internet en la función de marketing de las grandes empresas del municipio de Manizales durante el periodo de 1997 a 2001, el cual arrojó resultados determinantes para una toma de decisiones efectiva de aquellas organizaciones que deseen insertarse en la red.

La investigación incluye un análisis de tipo descriptivo de un total de 73 grandes empresas, según datos de la Cámara de Comercio de Manizales a 2001. Se escogieron las grandes empresas, porque comparadas con las medianas y pequeñas, son las que han realizado mayores inversiones de capital en tecnologías de información. Entre los principales aportes de la investigación están: a) Plantear recomendaciones a las empresas objeto de estudio para optimizar el uso de Internet como herramienta de marketing, b) Conocer, analizar y evaluar el uso de tecnologías Web, en el caso específico de Internet, como herramienta de apoyo a las estrategias de marketing de las organizaciones, durante 1997 a 2001, y c) Servir de referencia sobre la utilización de Internet en la función de marketing de las grandes empresas.

El referente teórico contiene dos elementos fundamentales: la conceptualización de grandes empresas y la función de marketing. Una empresa, en términos económicos, es una unidad que reúne los servicios de los factores de producción (recursos naturales, trabajo y capital) y los utiliza para producir bienes y/o servicios que vende a otras empresas, a las familias o al Estado. Según datos proporcionados por la Cámara de Comercio sede Manizales, dicha Institución clasifica las empresas según el régimen impuesto por el gobierno,(2) el cual define a la Gran Empresa como aquélla que: posee en su planta de personal más de 200 trabajadores y activos totales por más de quince mil (15.000) salarios mínimos mensuales legales vigentes. De acuerdo con lo anterior, los departamentos de Marketing se encuentran en una constante e intensa relación con sus

2 CONGRESO DE COLOMBIA. Op. cit.

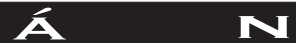

Universidad Autónoma de Manizales
$F$

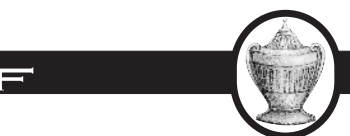

Año I3, Número 20, enero - junio 2006 
clientes y, aún, con sus competidores. La comunicación entre la tecnología y el Marketing se ha fusionado. Así, las organizaciones que incorporan tecnologías de información (TI) se enfrentan a una gran responsabilidad, que incluye comunicación a múltiples audiencias, entender los procesos de negocios de los clientes, enfocarse a las necesidades del cliente con sus procesos específicos y contextos tecnológicos y ofrecer soluciones que tienen requerimientos de integración corporativas.

El estudio es de tipo exploratorio y descriptivo, el cual fue aplicado a una muestra de 34 grandes empresas de un total de 73 ubicadas en la ciudad de Manizales. Se eligió esta muestra considerando en primera instancia que las empresas objeto de estudio, son aquéllas que tienen presencia en la red, sea a través de sitios Web o participación en negocios electrónicos. Las técnicas utilizadas fueron dirigidas a directores o jefes de departamentos de mercadeo.

A lo largo del estudio se explican las razones que progresivamente obligan a que las organizaciones apliquen tecnologías Web en sus actividades y estrategias de marketing. El análisis de los resultados obtenidos contiene información básica sobre Internet en las grandes empresas en términos de herramientas, actividades, estrategias y cambios producidos por Internet en la función de marketing. El $52,94 \%$ del total de las grandes empresas encuestadas pertenece al sector industrial, seguido de un $29,41 \%$ de las empresas que hacen parte del sector servicios y un $17,65 \%$ corresponde al sector comercial de la ciudad de Manizales.

De un total de 34 empresas encuestadas el $8.82 \%$ de ellas cuentan con Internet hace más de 5 años, siendo las pioneras en la instalación de este servicio. Para estas empresas los beneficios de Internet en la función de marketing están determinados por diferentes aspectos tales como el refuerzo de la imagen corporativa o también la realización de comercio electrónico con otras organizaciones. Es de anotar que un $44 \%$ de las empresas encuestadas aun no han implementado páginas Web en sus organizaciones, por razones como

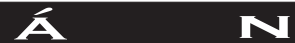

Universidad Autónoma de Manizales 
aumentos en costos administrativos, la falta de conocimiento de tecnologías de información, ausencia de información sobre las oportunidades que genera Internet para los negocios electrónicos y tradicionales.

De las 34 empresas encuestadas, se encontró que sólo 7 de ellas utilizan Internet como herramienta de publicidad, lo cual representa tan sólo el $20.6 \%$ del total, enfrentándose a un $64.7 \%$, en donde aun no tienen claridad sobre la pertinencia de la utilización de esta red de comunicación como una herramienta fundamental para dar a conocer su productos o servicios al mercado meta. Las acciones publicitarias más frecuentes son los banners, ya que cinco $(14,71 \%)$ de las grandes empresas las utilizan.

Las grandes empresas en Manizales, objeto de estudio, están realizando estrategias de marketing en la red y se están enfocando básicamente en establecer medios de atención al cliente pues según los resultados obtenidos mediante la encuesta, se encontró que del total de las empresas encuestadas el $58.82 \%$ consideran que la atención al cliente es la principal estrategia de marketing virtual, mientras que tan solo tres de ellas realizan pruebas de mercado que corresponden al $8.82 \%$ de las empresas entrevistadas.

En el sector de servicios las estrategias de marketing directo que más se aplican en Internet son: el servicio al cliente con un $40 \%$, el servicio post-venta (30\%) y la segmentación de mercados $(30 \%)$. Para el caso de las grandes empresas del sector comercial, las seis entrevistadas han tenido aumento de clientes gracias a la aplicación de Internet en la función de marketing, las principales razones que explican este aumento según los jefes y directores de departamentos de marketing son: a) La Posibilidad de llegar a más clientes ofreciendo mas productos sin salir de la casa, b) Aumento de la base de datos, hecho que les permite segmentar mejor su nicho de mercado, c) Posibilidad de tener acceso a tendencias del mercado que les permitan actuar más rápido, d) Presentar información a las personas de todos sus productos, y e)Se tiene mas contacto con las 
necesidades reales de sus clientes.

Para incrementar las posibilidades de éxito empresarial empleando Internet, se debe hacer una profunda reflexión sobre la empresa en la que habría que contemplar algunos aspectos como: a) Cómo se relaciona con sus clientes y proveedores, b) situación tecnológica en cuanto a Internet y sistemas de información y c) Conocimientos al respecto de las nuevas tecnologías de las personas que integran la organización, entre otros aspectos.

Con el fin de aprovechar el uso de la tecnología web en la función de marketing, se proponen los diseños de la estructura básica del nuevo negocio electrónico. En primera instancia realizar un autodiagnóstico. Después de ello habrá que concentrarse en el segundo paso: Revirtiendo la Cadena de Valor, el cual consiste en una transformación del modelo tradicional de la cadena de valor. Una empresa para llevar a cabo el nuevo modelo de negocio debe tomar en cuenta la redefinición exhaustiva de la cadena de valor tradicional y del poder innegable de las necesidades del mercado. El tercer y último paso es: La Elección de un Enfoque Estrecho. Debido a que no cualquier empresa es capaz de hacer muchas cosas bien al mismo tiempo, y debe focalizarse hacia excelencia en el servicio, excelencia operacional o excelencia en innovación continua.

Por tanto se necesita de una estrategia que afecte a las distintas partes de la cadena de valor, fomentar la política de crear valor al cliente, tanto en los departamentos como en la relación con otras empresas buscando mejoras en sus procesos de negocio que afectan a los clientes, una disminución de costos, motivarles en el cumplimiento de los objetivos, para ello es importante realizar contratos flexibles con terceras empresas, que permitan realizar variaciones de forma periódica en concordancia con las circunstancias cambiantes del mercado, etc.

Una conclusión final del estudio muestra que las grandes empresas en Manizales no tienen definida una estrategia de mix de marketing

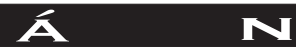

Universidad Autónoma de Manizales 
online. Están experimentando la red como medio de promoción, con mayor o menor criterio, interesadas en su mayoría en las posibilidades que se presume presenta la red como canal de distribución. El empleo del correo electrónico como medio para publicitar ciertos dominios, servicios o productos es cada vez más habitual. El e-mail marketing es un fenómeno cada vez más importante en Internet, como vía para contactar clientes de forma más directa. Se podría establecer un paralelo entre lo que es el marketing directo y el e-mail marketing siendo este último una técnica específica para Internet. Por esta razón se recomienda que los directivos deben diseñar estrategias en Internet que incentiven al cliente a visitar el sitio Web, buscando con ello sensibilizarlos sobre la utilización de que este medio les dará mayor facilidad para ahorrar tiempo y dinero teniendo resultados a un corto y mediano plazo.

FICHA DEL PROYECTO

\begin{tabular}{|c|c|}
\hline TÍTULO & $\begin{array}{l}\text { Estudio sobre los cambios generados por la } \\
\text { aplicación de Internet en la función de } \\
\text { marketing } \\
\text { de las grandes empresas del municipio de } \\
\text { Manizales }\end{array}$ \\
\hline INVESTIGADOR PRINCIPAL & Lina María Echeverri \\
\hline ESTUDIANTES PARTICIPANTES & $\begin{array}{l}\text { Miguel Ángel Maya } \\
\text { Juan David Mejía } \\
\text { Felipe García } \\
\text { Luis Eduardo García }\end{array}$ \\
\hline GRUPO DE INVESTIGACIÓN & Grupo de Estudio en Marketing - GEM \\
\hline LÍNEA DE INVESTIGACIÓN & Empresariado \\
\hline INSTITUCIÓN & Universidad Autónoma de Manizales \\
\hline TIPO DE PROYECTO & Exploratorio - Descriptivo \\
\hline CARÁCTER & Investigación aplicada \\
\hline
\end{tabular}

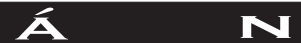

Universidad Autónoma de Manizales

\section{$\mathbf{R}$}

Año I3, Número 20, enero - junio 2006 


\section{INTRODUCCIÓN}

Hoy Internet es una herramienta necesaria para hacer negocios. Las empresas que incorporen el comercio electrónico, dentro de su estrategia empresarial podrán beneficiarse de nuevas oportunidades como por ejemplo, abrir el negocio a nuevos mercados, mejorar las relaciones con clientes y proveedores, simplificar la gestión de transacciones comerciales para ser eficaces y reducir costos. A pesar de que Internet es sólo un campo de pruebas para lo que será en un futuro muy cercano la autopista de la información, -que conectará el globo con una enorme capacidad de transmisión de datos a velocidades cada vez mayores- está cambiando lentamente la estructura informática y de mercadeo de las empresas.

\section{PROBLEMA DE INVESTIGACIÓN}

En Colombia, la utilización de Internet como herramienta de mercadeo se encuentra en una fase introductoria según el Informe Final de Conectividad Nacional.(3) De acuerdo con el informe, varias empresas han dirigido sus esfuerzos hacia Internet con el propósito de que éste se constituya como uno de sus principales puntos de venta, transformando la visión tradicional de negocio. Las ventajas de las nuevas tecnologías no están claras para algunas empresas. Por esta razón una de las principales estrategias de la Agenda Nacional de Conectividad es aumentar la competitividad de las empresas nacionales a través del uso y apropiación de las tecnologías de la información. Internet está pasando de ser un medio desconocido y mínimamente utilizado por las empresas hasta hace unos pocos años, a un nuevo canal de comunicación al que éstas se ven obligadas a trasladar una parte o el total de sus servicios si desean seguir siendo competitivas.

El décimo capítulo del informe de conectividad relacionado específicamente con Internet, señala una marcada diferenciación entre las grandes corporaciones y multinacionales, el sector

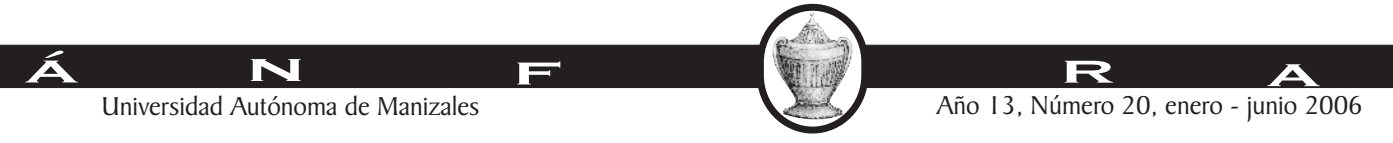


educativo de las principales universidades y grandes organismos estatales, y el sector productivo de la mediana y pequeña empresa. Las grandes corporaciones y multinacionales, acostumbradas a competir en el ámbito internacional y a estar a tono con los avances tecnológicos en el mundo, han adoptado rápidamente tecnología de punta para la comunicación e información. La experiencia y trayectoria que estas empresas han tenido en la implementación de sistemas de comunicación empresarial avanzados como redes de área local, sistemas de mensajería, sistemas de manejo empresarial (ERP), etc., hizo posible la rápida adopción y desarrollo de Internet como una herramienta de eficiencia y productividad.

Es importante que los empresarios consideren que Internet permite llegar no a un mercado masivo, sino a uno reiterativo, es decir cautivar al cliente de modo que regrese muchas veces a comprar el producto o el servicio. En el grupo de las grandes empresas, una meta clara en el corto plazo es orientar el mercadeo de sus productos directamente hacia el cliente, como soporte fundamental en su modelo de negocio: hacer al consumidor el centro de todas las acciones de la empresa. De acuerdo con lo anterior, Peter Clemente (1999) en su libro El Estado de la Net, concluye que: "El reto que muchas compañías enfrentan no es solo aprender cómo incorporar tecnología de Internet en sus prácticas empresariales, sino cómo entender su estructura y evolución." La utilización de Internet no es exclusiva del área de sistemas de las grandes empresas, éste tipo de tecnología de información debe incluir todas las áreas de la organización. Por lo tanto, a través de la función de marketing, las empresas tienen la posibilidad de acercarse más a su cliente de una forma directa y personalizada, como lo posibilita Internet.

La investigación propuesta busca identificar los cambios que se han generado por la aplicación de Internet en numerosos flujos de información y su efecto en las decisiones de mercado en las grandes empresas del municipio de Manizales. El estudio contempla los años de 1997 a 2001, un año después de que el primer proveedor de

3 BUSTAMANTE, Claudia Ximena y FAJARDO, Isabel Cristina. Op. cit.

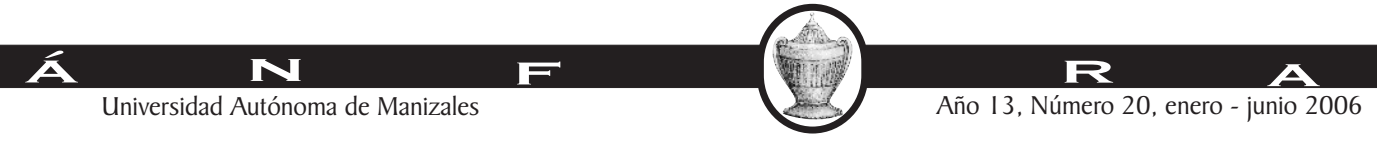


acceso al servicio de Internet llegara al municipio a través de la Red Interinstitucional de Manizales (RIM) en 1996; en Colombia Internet está desde 1991. De acuerdo con los objetivos planteados, se utilizó como técnica de investigación una entrevista, validada por juicio de expertos en las áreas de informática y mercadotecnia. La investigación incluye un análisis de tipo descriptivo de un total de 73 grandes empresas, según datos de la Cámara de Comercio de Manizales a 2001. Se escogieron las grandes empresas, porque comparadas con las medianas y pequeñas, son las que han realizado mayores inversiones de capital en tecnologías de información.

En Manizales, el uso de Internet en la función de marketing está muy poco desarrollado aún, las grandes empresas continúan aplicando estrategias apoyadas en el marketing tradicional, lo que lo hace muy interesante para su análisis. De acuerdo con la hipótesis anteriormente planteada, el alcance del proyecto fue a nivel local, el ámbito de trabajo fueron las grandes empresas, por esta razón los objetivos están orientados al diagnóstico evaluativo acerca de los cambios generados por la aplicación de Internet en los departamentos de marketing y a su vez, la proposición de estrategias y definición de herramientas que permitan obtener mayor provecho de la tecnología Web en la función de marketing de las empresas objeto de estudio.

\section{OBJETIVOS}

\section{OBJETIVO GENERAL}

Conocer y analizar los cambios generados por aplicación de Internet en la función de marketing de las grandes empresas del municipio de Manizales en el periodo de 1997 a 2001.

\section{OBJETIVOS ESPECIFICOS}

$\downarrow$ Detallar las formas e intencionalidad de la utilización de Internet por parte del departamento de marketing de las grandes empresas 
del municipio de Manizales.

$₫$ Conocer y describir las herramientas de Internet que soportan la función de marketing de las grandes empresas del municipio de Manizales.

$\varangle \quad$ Identificar y analizar las actividades de marketing que realizan las grandes empresas del municipio de Manizales.

$\varangle \quad$ Analizar las estrategias de marketing apoyadas en Internet de las grandes empresas del municipio de Manizales.

『 Conocer y evaluar los logros obtenidos por la aplicación de Internet en la función de marketing durante el periodo de 1997 a 2001 en términos de ventas, atención al cliente y uso de herramientas.

『 Proponer estrategias que permitan incursionar y mejorar la utilización de Internet en la función de marketing de las grandes empresas.

\section{RESULTADOS}

La aplicación de medios electrónicos e informáticos ha revolucionado la gestión de las empresas. Esta sección contiene el análisis de los resultados obtenidos a través de la investigación realizada sobre los cambios generados por la aplicación de Internet en la función de marketing de las grandes empresas de Manizales durante 1997 a 2001.

A lo largo del estudio se explican las razones que progresivamente obligan a que las organizaciones apliquen tecnologías Web en sus actividades y estrategias de marketing. De acuerdo con la población objeto de estudio, la actividad productiva del total de las empresas encuestadas está clasificada según el sector: 
Del total de las grandes empresas encuestadas 18 pertenecen al sector industrial, seguido de 10 de las empresas que hacen parte del sector servicios y seis corresponde al sector comercial de la ciudad de Manizales. Son empresas que en promedio llevan más de 30 años establecidas en la ciudad, y en su mayoría hacen parte del sector industrial, principal actividad económica del municipio.

1. Internet en las grandes empresas de Manizales

Las formas e intencionalidad de la utilización de Internet por parte del departamento de marketing de las grandes empresas del municipio de Manizales están determinadas inicialmente por el proceso de una lenta inserción de éstas compañías a la red de redes.

Según los resultados arrojados por la investigación se determinó que las grandes empresas de Manizales, tanto individuales como colectivas, están conectadas a Internet hace tres años en promedio, lo cual indica que este servicio es relativamente nuevo, claro está que de las 34 empresas encuestadas el $8.82 \%$ de ellas cuentan con Internet hace más de 5 años, siendo las pioneras en la instalación de este servicio. De acuerdo con lo anterior, es de considerar que el auge de la implementación de este servicio por parte de las empresas fue a comienzos del siglo XXI, donde el $29.41 \%$ de las empresas encuestadas implementaron este servicio, siendo este el porcentaje más alto.

Los resultados arrojados por la investigación, muestran que el $56 \%$ de las empresas objeto de estudio cuentan con sitio Web, lo que significa que están empezando a considerar la importancia del uso de creación de páginas Web en la época actual, donde el marketing y la tecnología se han fusionado, creando una interdependencia entre estos, con el fin de buscar presencia en la economía digital, tratando de pasar de un marketing de organización a un marketing más activo en un mercado cada vez más internacionalizado.

Para estas empresas los beneficios están determinados por

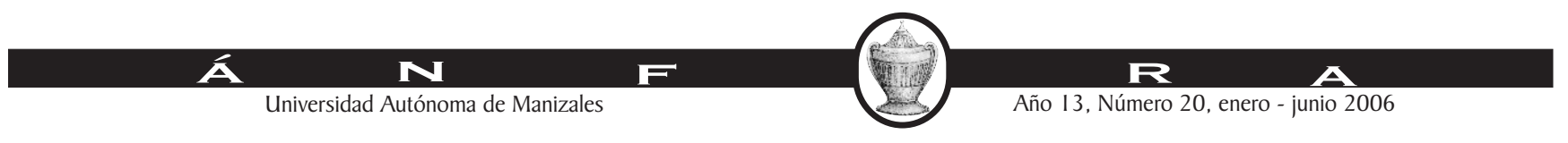


diferentes aspectos tales como el refuerzo de la imagen corporativa o también la realización de comercio electrónico con otras organizaciones. Es de anotar que un $44 \%$ de las empresas encuestadas aun no han implementado páginas Web en sus organizaciones, por razones como aumentos en costos administrativos, la falta de conocimiento de tecnologías de información, ausencia de información sobre las oportunidades que genera Internet para los negocios electrónicos y tradicionales.

Del total de las empresas encuestadas que cuentan con acceso a Internet, se detectó la amplia cobertura que tiene EPM en la región, prestando este servicio a 20 empresas encuestadas, seguido por Andinet el cual presta su servicio solo a cuatro empresas. Esta situación es causada principalmente porque EPM tiene una alianza estratégica con Emtelsa, empresa que presta el servicio de telefonía y comunicaciones en la capital Caldense.

Según la Figura 1, se puede observar que un $47 \%$ de las 34 empresas encuestadas cuentan con dominio o servidor propio, de igual forma un $44 \%$ de las empresas encuestadas cuenta con dominio o servidor alojado, y un $9 \%$ no cuenta con ningún tipo de servidor.

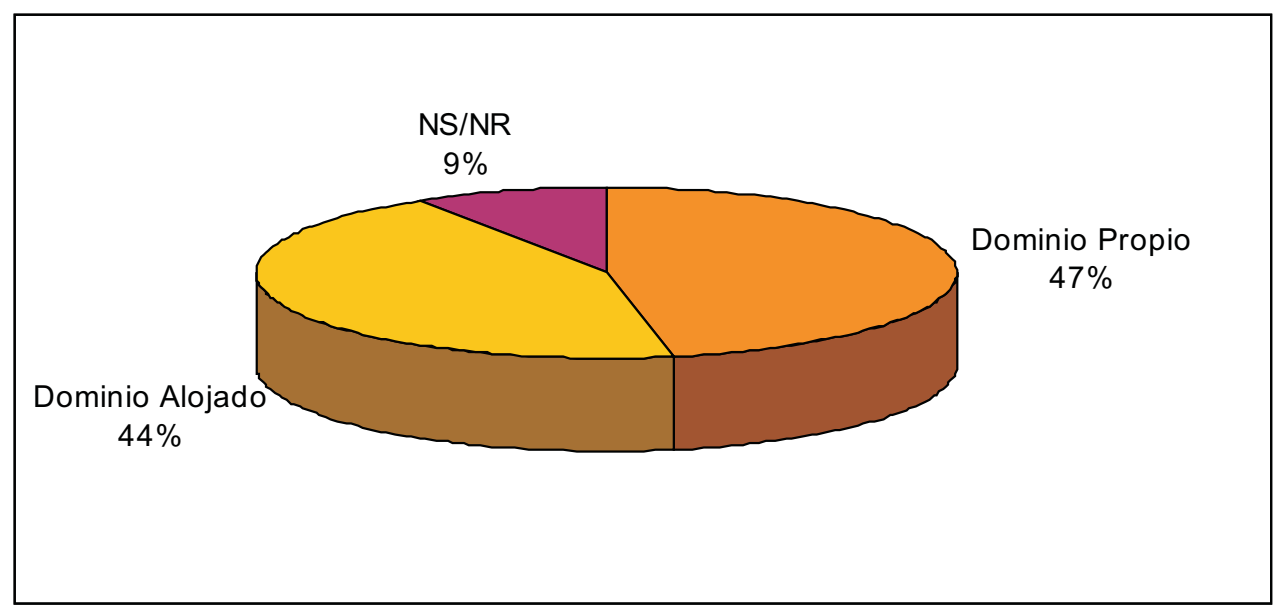

Figura 1. Tipo de Dominio de las grandes empresas de Manizales

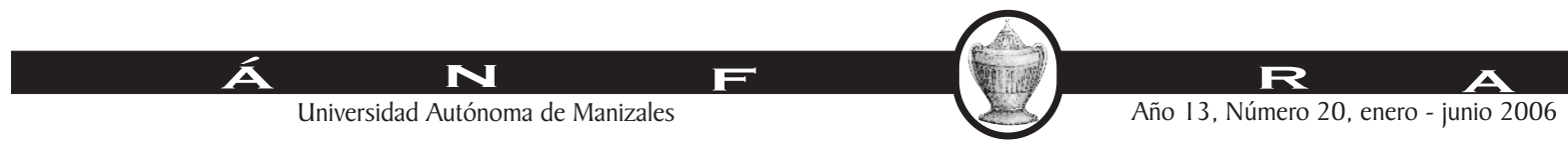


Según los datos arrojados por la investigación se encontró que las razones más importantes para que las empresas encuestadas mantengan una determinada presencia en Internet son: el refuerzo de su imagen corporativa y el ofrecimiento de bienes y servicios. Teniendo en cuenta que el $56 \%$ de estas empresas que mantienen una determinada presencia en Internet, está basada exclusivamente en la publicación de su sitio Web, y no en la realización del comercio electrónico, ampliación de su cuota de mercado y la identificación de necesidades y deseos de sus clientes potenciales, tal como lo afirman Vázquez y Tres palacios (1994): "El objetivo del marketing es tratar de conocer las necesidades genéricas del consumidor o carencias básicas propias de la naturaleza y condición humana".

\section{Herramientas de Internet en la función de marketing}

De las 34 empresas encuestadas, se encontró que sólo 7 de ellas utilizan Internet como herramienta de publicidad, lo cual representa tan sólo el $20.6 \%$ del total, enfrentándose a un $64.7 \%$, en donde aun no tienen claridad sobre la pertinencia de la utilización de esta red de comunicación como una herramienta fundamental para dar a conocer su productos o servicios al mercado meta.

Con un porcentaje menor de $14.7 \%$ se encuentran aquellas empresas donde los jefes de departamento desconocen si la empresa cuenta con publicidad en Internet. De acuerdo con la fundamentación teórica de la investigación se puede evidenciar que la tecnología es el motor de la nueva economía, permitiendo facilitar la circulación de la moneda: la información materializada en conocimiento. Pero hay otro factor para el que la tecnología también actúa como elemento potenciador: el de la producción de conocimiento con mayor agilidad y eficiencia considerando la Internet como una herramienta indispensable.

De acuerdo con lo mencionado, se infiere que el sector industrial es en donde más se percibe el impacto de Internet, no sólo como herramienta de comunicación interna, sino también como un medio

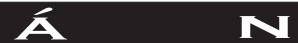

Universidad Autónoma de Manizales 
para darse a conocer a su mercado meta. Las grandes empresas tanto comerciales, como industriales y de servicios deben considerar que la Era de la Información ha llegado y ha evolucionado los procesos de todos los negocios en la cual puede dar facilidad para intercambios comerciales y las diferentes formas de $E$-Business.

A través de la encuesta realizada se encontró que el modelo de publicidad on-line más utilizado en Internet, es el banner, con una participación del $8.8 \%$, seguido por la Sponsorización con una participación de 5.9\%, y por último, Patrocinio y Product-Placement con una participación de $2.9 \%$ cada una. El porcentaje más significativo corresponde a un $79,4 \%$ del total de las empresas encuestadas las cuales no conocen o no tienen información sobre los diferentes modelos de publicidad on-line.

La publicidad puede estar apoyada por las tecnologías de información, entre las cuales, una de las más importantes es Internet y ha pasado de ser un medio de comunicación masiva a constituirse

\begin{tabular}{|l|c|c|c|c|c|c|}
\hline \multirow{2}{*}{ Razones } & \multicolumn{6}{|c|}{ Orden de Importancia } \\
\cline { 2 - 7 } & $\begin{array}{c}\text { Primer } \\
\text { lugar }\end{array}$ & $\begin{array}{c}\text { Participación } \\
\text { porcentual }\end{array}$ & $\begin{array}{c}\text { Segundo } \\
\text { lugar }\end{array}$ & $\begin{array}{c}\text { Participación } \\
\text { porcentual }\end{array}$ & $\begin{array}{c}\text { Tercer } \\
\text { lugar }\end{array}$ & $\begin{array}{c}\text { Participación } \\
\text { porcentual }\end{array}$ \\
\hline $\begin{array}{l}\text { Reforzar imagen } \\
\text { corporativa }\end{array}$ & 12 & $35.29 \%$ & 3 & $8.82 \%$ & 5 & $14.71 \%$ \\
\hline $\begin{array}{l}\text { Ofrecer información } \\
\text { sobre bienes y } \\
\text { servicios }\end{array}$ & 7 & $20.59 \%$ & 10 & $29.41 \%$ & 3 & $8.82 \%$ \\
\hline Interés publicitario & 2 & $5.88 \%$ & 2 & $5.88 \%$ & 5 & $14.71 \%$ \\
\hline $\begin{array}{l}\text { Asistencia a los } \\
\text { consumidores y } \\
\text { usuarios }\end{array}$ & 3 & $8.82 \%$ & 7 & $20.59 \%$ & 4 & $11.76 \%$ \\
\hline $\begin{array}{l}\text { Ventas y comercio } \\
\text { electrónico }\end{array}$ & 2 & $5.88 \%$ & 1 & $2.94 \%$ & 4 & $11.76 \%$ \\
\hline Comunicación interna & 2 & $5.88 \%$ & 1 & $2.94 \%$ & 2 & $5.88 \%$ \\
\hline $\begin{array}{l}\text { Información } \\
\text { financiera }\end{array}$ & 0 & $0,00 \%$ & 1 & $2.94 \%$ & 1 & $2.94 \%$ \\
\hline $\begin{array}{l}\text { Adquirir base de } \\
\text { Datos }\end{array}$ & 0 & $0,00 \%$ & 4 & $11.76 \%$ & 1 & $2.94 \%$ \\
\hline Ampliar su mercado & 2 & $5.88 \%$ & 2 & $5.88 \%$ & 3 & $8.82 \%$ \\
\hline \multicolumn{1}{|c|}{ TOTAL } & $\mathbf{3 0}$ & $\mathbf{1 0 0 \%}$ & $\mathbf{3 1}$ & $\mathbf{1 0 0 \%}$ & $\mathbf{2 8}$ & $\mathbf{1 0 0 \%}$ \\
\hline
\end{tabular}

Tabla 1. Razones de las grandes empresas para estar presente en Internet

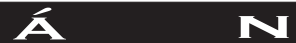

Universidad Autónoma de Manizales

\section{$\mathbf{R}$}

Año I3, Número 20, enero - junio 2006 
en el mecanismo de apoyo interactivo entre las empresas y sus clientes a través de la utilización de la investigación de mercados.

En la Figura 3 se puede observar que en el sector Comercial la principal estrategia de comunicación es la televisión con un porcentaje del 70\%; seguido del sector Industrial con un $62,5 \%$ representado por muestras, ferias y eventos; y por último en el sector servicios la principal estrategia de comunicación fue la de catálogos, con un porcentaje de utilización de $31 \%$ con respecto a otros medios.

3. Descripción de las actividades de marketing de las grandes empresas de Manizales

Las actividades de marketing se hacen cada vez más complicadas para las empresas. El paso del tiempo está poniendo de relieve que conocer a los consumidores es progresivamente más difícil, pues los mercados se especializan cada vez más. Los mercados ya no son masivos. Los productos eran más homogéneos y el comportamiento de los consumidores más uniforme. Las campañas de comunicación,

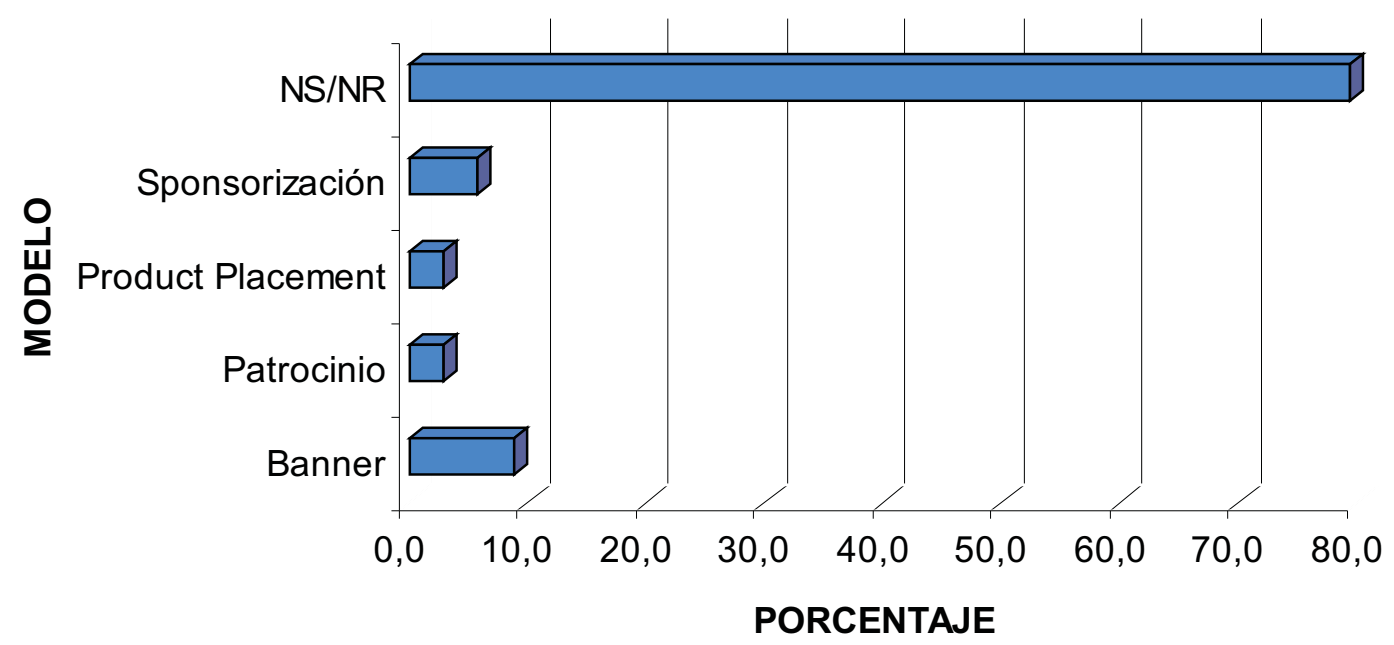

- Figura 2. Modelos de Publicidad On-line utilizados por las grandes empresas de Manizales 
también eran más efectivas. Con la irrupción de nuevas tecnologías permiten obtener y tratar una gran cantidad de información.

La publicidad, el comercio electrónico, los mailings personalizados, el marketing directo, la oferta de los operadores y las redes internas como intranets son instrumentos fundamentales para conocer los perfiles demográficos, sociográficos y sicográficos de los consumidores, es decir, sus características relativas a edad, sexo, status profesional, cultura, renta o personalidad y estilos de vida.

Como se puede observar en la Tabla 2, las grandes empresas de los sectores productivos concentran sus actividades de marketing en publicidad $(29,41 \%)$, mailings personalizados $(38,24 \%)$, marketing directo $(32,35 \%)$ y documentos informativos $(38,24 \%)$ en la red. En el sector servicios, la actividad de marketing más utilizada son los mailings personalizados. En el sector industrial, las actividades de marketing más utilizadas son el marketing directo y personalizado. Y en el sector comercial, la actividad de marketing empleada son los

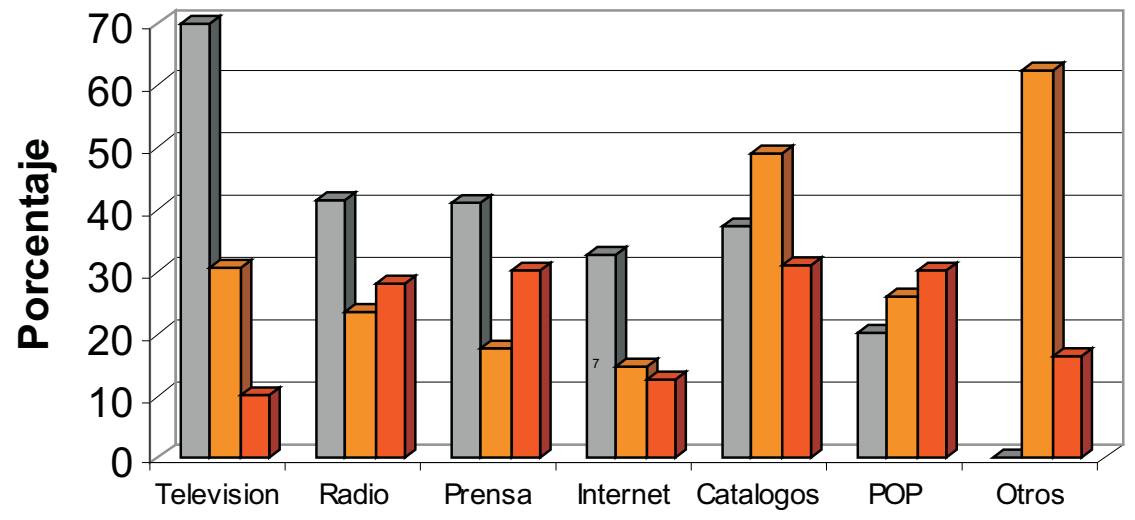

Estrategias

$\square$ Comercial $\square$ Industrial $\square$ Servicio

- Figura 3. Estrategias de Comunicación utilizadas por las Grandes Empresas según sector productivo 
documentos informativos.

Las acciones publicitarias más frecuentes son los banners, ya que cinco $(14,71 \%)$ de las grandes empresas lo usan para su publicidad, seguido del e-mail, usado por el 100 por ciento, lo que demuestra que estas empresas son conservadoras y usan herramientas que ya conocen y saben como funcionan.

El total de las empresas consultadas, consideran que Internet no es un mercado maduro, aunque también lo ven como un problema a la hora de anunciarse por el elevado costo y la baja presencia del PC en los hogares, por lo que estas empresas siguen confiando más en la publicidad tradicional.

El reto futuro para estas empresas es aumentar la creencia en la red como canal de distribución y comunicación, ya que los

\begin{tabular}{|c|c|c|c|c|c|c|c|}
\hline \multirow{2}{*}{$\begin{array}{l}\text { Actividades de } \\
\text { Marketing }\end{array}$} & \multicolumn{2}{|c|}{ Sector Comercial } & \multicolumn{2}{|c|}{ Sector Industrial } & \multicolumn{2}{|c|}{ Sector Servicios } & \multirow{2}{*}{$\begin{array}{c}\text { Total } \\
N^{\circ} \text { de } \\
\text { empresas }\end{array}$} \\
\hline & $\begin{array}{c}N^{\circ} \text { de } \\
\text { empresa }\end{array}$ & $\%$ & $\begin{array}{r}\mathrm{N}^{\circ} \mathrm{de} \\
\text { empres }\end{array}$ & $\%$ & $\begin{array}{r}N^{\circ} \mathrm{de} \\
\text { empres }\end{array}$ & $\%$ & \\
\hline Publicidad & & & 7 & 70 & 3 & 30 & 10 \\
\hline Comercio electrónico & 1 & 16,67 & 4 & 66,67 & 1 & 16,67 & 6 \\
\hline Noticias & & & 3 & 60 & 2 & 40 & 5 \\
\hline $\begin{array}{l}\text { Mailings } \\
\text { Personalizados }\end{array}$ & 3 & 23,08 & 5 & 38,46 & 5 & 38,46 & 13 \\
\hline $\begin{array}{l}\text { Desarrollo de páginas } \\
\text { Web }\end{array}$ & & & 2 & 40 & 3 & 60 & \\
\hline $\begin{array}{l}\text { Marketing directo y } \\
\text { personalizado }\end{array}$ & & & 8 & 72,73 & 3 & 27,27 & 11 \\
\hline Webmarketing & & & 2 & 66,67 & 1 & 33,37 & 3 \\
\hline Informativos & 2 & 15,38 & 5 & 38,46 & 6 & 46,15 & 13 \\
\hline $\begin{array}{l}\text { Sistemas de } \\
\text { información integrados } \\
\text { a E-commerce }\end{array}$ & & & 1 & 100 & & & \\
\hline Intranet & 1 & 20 & 2 & 40 & 2 & 40 & 5 \\
\hline NS/NR & 1 & 25 & 2 & 50 & 1 & 25 & 4 \\
\hline TOTAL & 8 & $100 \%$ & 41 & $100 \%$ & 27 & $100 \%$ & 70 \\
\hline
\end{tabular}

Tabla 2. Actividades de Marketing de las grandes empresas por sector productivo.

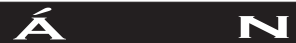

Universidad Autónoma de Manizales

\section{$\mathbf{R}$}

Año 13, Número 20, enero - junio 2006 
entrevistados no la considera como tal canal y algunos de los gerentes y jefes de departamento de mercadeo de las grandes empresas ve Internet "como una inversión de cara al futuro", opiniones "influidas por el mal momento que atraviesa el sector"

Internet se ha constituido en el cuarto canal de comunicación, donde el naciente marketing virtual no sólo ha establecido nuevas reglas de juego, sino que ha ayudado al marketing tradicional a conocer más a fondo a sus clientes.

4. Estrategias de marketing apoyadas en Internet de las grandes empresas

Es importante saber que Internet sirve para dirigir la información a los nichos de mercado para productos específicos. De acuerdo con lo mencionado anteriormente, la información recogida por el instrumento utilizado en la presente investigación señala que las estrategias de marketing apoyadas o no en Internet están estrechamente relacionadas con el sector productivo al que la empresa pertenece.

Es de destacar que la mayoría de las empresas del municipio de Manizales están clasificando su producto dentro del ramo o segmento de bienes de consumo, viéndose reflejado que 25 empresas de las 34 encuestadas en el municipio están orientando su producto o servicio hacia esta categoría.

El diseño de estrategias varía en el marketing convencional y en el marketing virtual, principalmente porque el contacto con el cliente es totalmente diferente. La interactividad, la personalización y la transnacionalidad son las principales ventajas del marketing electrónico frente al convencional de acuerdo con los planteamientos de Robert Plant (2001).

Al analizar los datos arrojados por la encuesta, se encontró que para algunas de las empresas la estrategia de marketing virtual



Universidad Autónoma de Manizales 
más utilizada actualmente es la atención al cliente como se observa en la Tabla No 3

Las grandes empresas en Manizales, objeto de estudio, están realizando estrategias de marketing en la red y se están enfocando básicamente en establecer medios de atención al cliente pues según los resultados obtenidos mediante la encuesta, se encontró que del total de las empresas encuestadas el $58.82 \%$ consideran que la atención al cliente es la principal estrategia de marketing virtual, mientras que tan solo tres de ellas realizan pruebas de mercado que corresponden al $8.82 \%$ de las empresas entrevistadas.

De acuerdo a los datos obtenidos, encontramos que las principales estrategias de marketing aplicadas por las grandes empresas del municipio de Manizales son respectivamente:

$₫ \quad$ La atención al cliente con $58.82 \%$ del total de las empresas. Este servicio permite consolidar los clientes actuales atendiendo reclamos y sugerencias. Gran parte de las estrategias del mercadeo directo consisten en llevar a la empresa hacia el cliente. Este servicio permite integrar todos los canales de comunicación y de servicio al cliente en bases de información organizada y accesible, y crear una relación más personalizada y provechosa con cada uno de sus clientes. En el mundo actual influido por la tecnología y orientado a

\begin{tabular}{|c|c|c|}
\hline Estrategias & Número de Respuestas & Participación Porcentual \\
\hline Investigación de Mercados & 11 & $32,35 \%$ \\
\hline Lanzamiento & 10 & $29,41 \%$ \\
\hline Comportamiento & 6 & $17,65 \%$ \\
\hline Inteligencia Comercial & 7 & $20,59 \%$ \\
\hline Pruebas de Mercadeo & 3 & $8,82 \%$ \\
\hline Posicionamiento & 7 & $20,59 \%$ \\
\hline Atención al Cliente & 20 & $58,82 \%$ \\
\hline Post-Venta & 13 & $38,24 \%$ \\
\hline Segmentación & 8 & $23,53 \%$ \\
\hline
\end{tabular}

Tabla 3. Estrategias de marketing en Internet de las Grandes empresas de Manizales

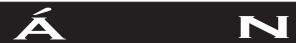

Universidad Autónoma de Manizales 
los servicios, los clientes se inclinan hacia un mayor nivel de servicio personal, así como hacia las ofertas de servicios y productos a medida. Para satisfacer esto, se necesita tener la capacidad de conocer las necesidades de los clientes de forma más detallada y responder más rápidamente que antes. Por último, las relaciones con los clientes a largo plazo son la clave para la rentabilidad de la empresa. Estas relaciones requieren satisfacer las necesidades y deseos del cliente mejor que la competencia.

¿ El servicio post-venta con $38.24 \%$ del total de las empresas. Permite adquirir información a la empresa a través de Internet para el cliente estar informado de todos los cambios, catálogo de productos, precios. Así mismo Internet permite a las empresas reducir coste de comunicación al mismo tiempo que ofrece servicio de valor añadido y orientación a los clientes (reclamos, sugerencias y garantías). Se debe atender a las necesidades del consumidor y no limitarse a venderle simplemente un producto.

『 La investigación de mercados con $32.35 \%$ del total de las empresas. El desarrollo de las nuevas tecnologías y especialmente con la utilización generalizada de Interne, las decisiones de compra cambian notablemente. Se realiza para aumentar el mercado, llegar a más clientes y/o vender más productos o servicios a los mismos consumidores. A medida que las empresas aumentan su mercado (nacional e internacional) la necesidad de información sobre los mercados es mayor porque los mercados cada vez son más grandes y más distantes. Como la competencia se ha vuelto más intensa, los empresarios necesitan información oportuna, ya que el entorno cambia con rapidez. La investigación de mercados es la herramienta más utilizada para tomar decisiones de negocio y para conocer más profundamente al consumidor y sus necesidades.

$\downarrow \quad$ El lanzamiento de productos (nuevos y existentes) con $29.41 \%$ del total de las empresas. La red permite dar a conocer los productos y servicios de las empresas de manera detallada y precisa respectivamente.

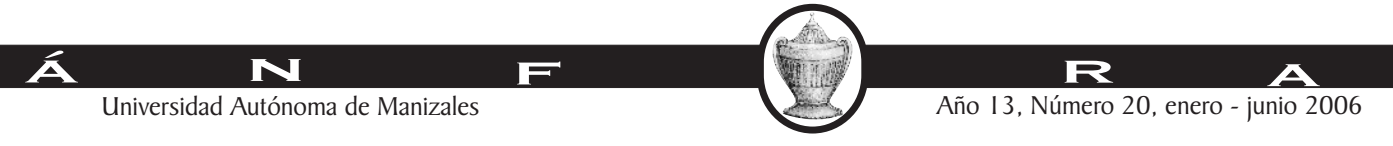


5. Cambios y logros obtenidos por la aplicación de Internet en la función de marketing durante el periodo de 1997 a 2001

Para la mayoría de las grandes empresas encuestadas el aumento de clientes por la aplicación de Internet en la función de marketing no ha sido tan significativo, a diferencia de aquellas organizaciones que pertenecen al sector comercial. Para el caso de las grandes empresas del sector comercial, 6 (seis) de ellas han tenido aumento de clientes gracias a la aplicación de Internet en la función de marketing, las principales razones que explican este aumento según los jefes y directores de departamentos de marketing son:

\& La Posibilidad de llegar a más clientes ofreciendo más productos sin salir de la casa.

$\checkmark$ Aumento de la base de datos, hecho que les permite segmentar mejor su nicho de mercado.

$\downarrow$ Posibilidad de tener acceso a tendencias del mercado que les permitan actuar más rápido.

$\downarrow$ Presentar información a las personas de todos sus productos.

$₫$ Se tiene mas contacto con las necesidades reales de sus clientes.

Por otro lado estas empresas consideran que los logros obtenidos por la inserción de sus productos en Internet han sido satisfactorios, ya que con éste, han logrado agilizar los trámites en cuanto a pedidos y reservas. Otro de los logros es brindar información a la población residente en el exterior quienes avivan su sentido de patria cuando se encuentran lejos de ésta.

Continuando con el análisis se puede observar, que las empresas comerciales presentan una desventaja con respecto a los demás sectores que pone en evidencia a las empresas manizaleñas a no reconocer la oportunidad de crecer y expandirse a través de esta tecnología Web. Como se mencionó en el referente teórico, es necesario cambiar la forma de hacer negocios, pasando del mercadeo convencional hacia el mercadeo directo, con el cual lograremos llegar 
a los clientes en una forma más directa y en tiempo real. Por otro lado hemos encontrado que las empresas industriales son quienes más se han interesado en mercadear sus productos por Internet como lo menciona Stanton y Etzel (2000): "Solo aquellas empresas que se proyecten a un futuro podrán servir a sus clientes en la forma que ellos deseen".

5.1 Ventajas y desventajas del Marketing por Internet. Las principales ventajas y desventajas que las grandes empresas encuentran en el marketing por Internet se presentan en el siguiente cuadro:

\begin{tabular}{|ll|}
\hline Sector & \multicolumn{1}{c|}{ Ventajas } \\
\hline & Reducción de gastos en publicidad tradicional. \\
& Creación de novedosos catálogos. \\
& Atención las 24 horas del día. \\
& Mayor cobertura de mercados. \\
& Posicionamiento de la empresa. \\
& Mayor rapidez en intercambio de datos. \\
& Mayor comodidad para el cliente. \\
& Recolección de información de mercado en tiempo real \\
& Actualización constante de los productos ofrecidos. \\
& Rapidez. \\
& Bajo costo. \\
& Amplia difusión. \\
& Ampliación de cobertura. \\
& Estar al día en tendencia de mercados. \\
& Mayor agilidad para contactar clientes y proveedores. \\
& Comercialización con otros países. \\
& Menos dificultad con el cambio de idioma. \\
& Posicionamiento. \\
\hline Industrial & Ágil, Rápido, concreto. \\
& Llegar a más clientes. \\
Reducción de costos. \\
Manejo de estrategias puntuales. \\
Ser más competitivo \\
Ágil, Rápido, concreto. \\
Llegar a más clientes. \\
Reducción de costos. \\
Manejo de estrategias puntuales. \\
\hline Servicios
\end{tabular}

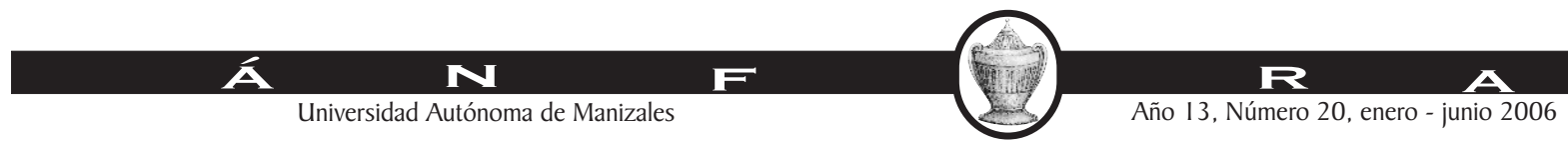




\begin{tabular}{|ll|}
\hline Sector & \multicolumn{1}{c|}{ Desventajas } \\
\hline \multirow{3}{*}{ Comercial } & Falta de desarrollo tecnológico para el ingreso a Internet \\
& Bajo nivel de presencia de transacciones en la red por bajo nivel de \\
& confianza. \\
& Alto nivel de competencia. \\
\hline \multirow{3}{*}{ Industrial } & Distribución del Producto. \\
& Aumento en el uso de aplicación y tecnología. \\
& Falta de generación de cultura de compra por Internet. \\
& Falta de conocimiento en el manejo por parte de las empresas. \\
\hline \multirow{3}{*}{ Servicios } & Requiere mucha publicidad. \\
& No todas las personas tienen acceso a este medio. \\
& No existe contacto directo con el cliente. \\
& Falta de seguridad en la red. \\
\hline
\end{tabular}

Es de anotar que las grandes empresas, específicamente del sector industrial son las que más ventajas encuentran en la aplicación de Internet en la función de marketing, sin embargo, son las que menos esfuerzo han dedicado en volver sus sitios Web interactivos y productivos.

\section{Proposición de estrategias de marketing basadas en la tecnología}

Web: Internet

Para incrementar las posibilidades de éxito empresarial empleando Internet, se debe hacer una profunda reflexión sobre la empresa en la que habría que contemplar aspectos como:

d Cómo se relaciona con sus clientes y proveedores.

d Estudio de sus procesos.

$\downarrow$ Situación tecnológica en cuanto a Internet y sistemas de información.

$\boldsymbol{d}$ Conocimientos al respecto de las nuevas tecnologías de las personas que integran la organización.

¿ Cuáles son los planes de futuro de la empresa.

$\mathbb{\Phi}$ Cuáles son sus ventajas competitivas respecto a la competencia.

Una de las principales metas de la función de marketing de las grandes empresas, va más allá de aumentar su participación en el mercado, se enfoca en incrementar el consumo de sus clientes tradicionales y

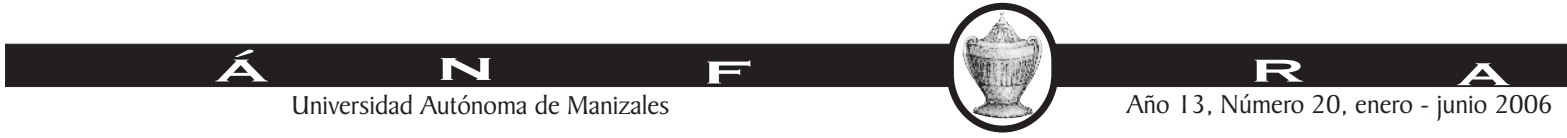


potenciales. Esto se puede lograr, si la empresa considera como alternativa insertarse a Internet teniendo en cuenta lo siguiente:

¿ Que los clientes potenciales lleguen al sitio Web.

d Que los que llegan, compren.

¿ Que los que compran, vuelvan.

El mercadeo de la empresa en Internet es un mercadeo a distancia. No se trata a los clientes personalmente. Todo lo que puede hacer la empresa para mejorar sus ventas es, de una parte, ajustar sus herramientas, $y$, de otra, ejecutar acciones que provoquen comportamientos favorables para que esa población dispersa se constituya en su clientela virtual. La empresa tiene que administrar las relaciones con esa clientela a través de herramientas tecnológicas, soportadas en investigaciones o inteligencia de mercados. Para lograr esto la empresa debe seguir los siguientes pasos para identificar el mercado:

1. Elaborar el perfil del mercado al que se quiere llegar.

2. Decidir el enfoque que se le dará al sitio Web por medio del contenido y las imágenes dentro del mismo.

3. Identificar las características de las personas que visitarán el sitio Web de la empresa, es decir, aquellas personas que son compradores potenciales.

4. Decidir los servicios que deberán ofrecerse para satisfacer las necesidades del nicho de mercado definido e incluirlas en los procesos de atención al cliente.

5. Determinar herramientas de retroalimentación, para asegurarse que los consumidores realmente están satisfechos o para identificar oportunidades de mejora (para lo cual será necesario utilizar herramientas dentro del sitio como grupos de discusión, cuestionarios, llamadas telefónicas de seguimiento, etc.)

Se hace necesario por tanto adaptarse a los cambios, a veces

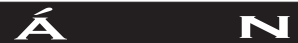

Universidad Autónoma de Manizales 
llegará con pequeñas modificaciones, pero en otras ocasiones será necesario incluso el replantearse el modelo de negocio, y para asegurarse que hacemos lo correcto debemos conseguir información sobre los clientes y hacer un uso inteligente de la misma.

Por esta razón, hay una gran posibilidad de intermediación, que permita fortalecer la aplicación de Internet en la función de marketing de las grandes empresas del sector comercial de la ciudad de Manizales. Se recomienda desarrollar el siguiente plan de mercadeo en su sitio Web:

『 Presentar su catálogo online. Esto es lo mínimo que puede ofrecer un distribuidor online, listando en su catálogo sus productos, mostrando fotos, descripciones, y presentando precios, para que luego los clientes los encarguen por teléfono o personalmente.

『 Procesar los pedidos de compra online. Algunas empresas comerciales permiten al cliente armar una canasta de compra para que luego completen la transacción telefónicamente. La posibilidad de completar la orden telefónicamente se debe en parte a que muchos consumidores desconfían de enviar la información de su tarjeta de crédito por Internet por miedo a que sea interceptada por terceros y usada fraudulentamente. Es importante considerar que la sociedad manizaleña si bien tiene acceso a Internet, por desconocimiento y desconfianza no son grandes compradores a través de la Web.

$\varangle \quad$ Procesar las transacciones monetarias online. LOS comercios electrónicos avanzados poseen sistemas con los cuales reciben la información de pago del cliente por Internet y procesan esta compra electrónicamente. El envío de esta Información debe estar protegida y luego se envía el producto por el correo tradicional. Para eso la empresa debe tener un buen sistema de logística. 


\section{$\downarrow \quad$ Completar la transacción, enviando el producto online.}

Cuando la compra es de un producto de contenido intangible, como son el software o la música, éste puede ser enviado por Internet, evitando un envío a través del correo.

Para aquellas empresas pertenecientes al sector servicios de la ciudad de Manizales que fueron objeto de estudio de la presente investigación, es de considerar que éste es un sector naciente en la economía local, por esta razón con la utilización de las nuevas tecnologías, han aparecido sistemas y técnicas que facilitan el obtener "conocimiento" de los clientes, a través de la recopilación de datos de los distintos departamentos o procesos de negocio que intervienen o se relacionan con los clientes, lo que refuerza a los métodos tradicionales de llegar a los clientes, como mailings, cartas personalizadas, visitas comerciales, etc. Por otro lado se debe aprovechar nuevas herramientas como el marketing "uno a uno" a través de Internet o nuevos sistemas de recompensas y fidelización de clientes, con el objetivo de conseguir la mayor cantidad de información posible sobre los clientes, usuarios o visitantes, para así, poder ofrecer el mayor valor posible.

Es de anotar, que el sector servicios tiene una ventaja competitiva y comparativa con el resto de los sectores. Estas grandes empresas de la ciudad de Manizales han implementado la red de Intranet, a través de la cual administran información de uso interno de la compañía. En cuanto a la aplicación de Internet en la función de marketing, este tipo de empresas puede aprovechar en primer lugar que se encuentran en un ciclo de vida introductoria, que exige el uso de tecnologías de información de avanzada soportada en las tecnologías Web de Intranet, Extranet e Internet. Por esta razón, las grandes empresas de la ciudad de Manizales que ofrecen servicios pueden optar por desarrollar un programa de fidelización de clientes (marketing Uno a Uno). Este programa debe recoger información sobre:

$\downarrow \quad$ Segmentación del mercado (actualizado).

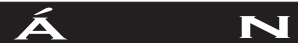

Universidad Autónoma de Manizales 
$\$ \quad$ Listado de clientes más importantes o de mayor valor.

$₫ \quad$ Porcentaje de influencia en las utilidades de la compañía.

$\downarrow$ Expectativas de los clientes con respecto a los servicios existentes.

$\downarrow$ Sugerencias que los clientes han formulado en el último año, cuya solución haya permitido diferenciar el servicio.

『 Lista de variables que inciden en la decisión de compra de los clientes por servicio.

$\downarrow \quad$ Lista de variables relacionadas con la valoración del servicio por parte de los clientes.

『 Políticas de confidencialidad, actualización y mantenimiento de datos relacionadas con los clientes.

A la hora de instrumentar la interacción con los clientes (además del propio sitio Web de la empresa), el programa de fidelización, servirá para mejorar la relación con los clientes, retenerles y motivarles a que compren más. El método de fidelización, consiste básicamente en propiciar que cada cliente incremente sus compras, acumulando de esta manera puntos que luego canjeará por productos, o bien descuentos en el acto. Para ello, se emiten cupones acumulables (puntos) o se confecciona una tarjeta personal (descuentos).

De las dos alternativas, una de ellas (la tarjeta con los datos del usuario) posibilita a la empresa no sólo premiar a sus clientes y motivarles a comprar, sino conocer mejor sus hábitos de consumo y de compra, determinar qué servicios productos se asocian a otros, y la variación de tendencias sobre volumen facturado. Sumado a lo anterior, de un total de diez empresas del sector servicios objeto de estudio, nueve grandes empresas no tienen aún establecido un plan de marketing relacional, donde se propicie a que clientes eventuales se conviertan en asiduos.

Sólo una empresa ya tiene adelantado dicho programa cuya actividad productiva está relacionada con las telecomunicaciones. Esta situación se da porque se han confiado de ser las únicas

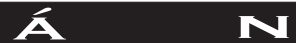

Universidad Autónoma de Manizales 
empresas que ofrecen servicios donde la competencia es prácticamente nula. Aun no se han percatado de que en muy poco tiempo llegarán nuevos competidores en el sector eléctrico y de las telecomunicaciones que podrían afectar el comportamiento de su mercado objetivo.

Un componente fundamental en el programa de fidelización que se sugiere, es la creación de un centro de atención telefónica (callcenter), ya que permite conocer las quejas e inquietudes de los clientes y darles pronta solución. Estará constituido por un personal especializado que recibe la llamada y resuelve el problema (interactuando a veces con otros departamentos), canaliza sugerencias que sirven para mejorar, modificar o complementar el servicio brindado, promocionan ofertas y nuevas aplicaciones generando valor agregado.

Pero un servicio de este tipo no se reduce a un conjunto de líneas telefónicas con varios operadores, las empresas deben tener base de datos completa, que almacene todos los datos significativos de cada cliente, conjuntamente con los reclamos efectuados y su solución. Adicionalmente la empresa debe tener instalaciones que garanticen un tiempo de espera mínimo. El operador, al recibir una llamada, debe, de forma breve, enterarse de la situación del cliente, a fin darle un trato personalizado.

\section{CONCLUSIONES}

En la actualidad, Internet pasa de ser uno de los múltiples medios de comunicación de masas, a convertirse en el medio de comunicación por excelencia. A finales de los 90, y a las puertas del siglo XXI, son ya miles las empresas que se han dado cuenta de esto y que, ya disponen de Web en Internet. Una de las ventajas de Internet sobre otros medios, es la capacidad de saber exactamente cuánta gente ve un anuncio digital o "banner". Lo que se mide son las impresiones o cantidad de veces que el anuncio es desplegado. Adicionalmente, se puede saber la cantidad de veces que los lectores no sólo vieron el

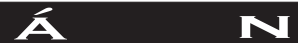

Universidad Autónoma de Manizales 
anuncio, sino que hicieron "clic" sobre el mismo.

No existe un modelo de gestión empresarial claro en Internet. Podemos destacar la falta de "históricos" debido a la inmadurez de Internet y la falta de profesionales que dominen completamente las estrategias de la red pero, sobre todo, la "revolución" que produce en la empresa, la mezcla de sus negocios tradicionales con los electrónicos y el cambio cultural que esto implica.

En el mundo empresarial se exigirá de una gerencia cada vez más plana, (y mucho menos vertical) con cada vez más y más información producida e intercambiada digitalmente a fin de descubrir las diferentes necesidades en las compañías y sus clientes.

La efectividad de los gerentes de mercadeo de las compañías, dependerá de lo creativos que sean en el manejo de la información y de la tecnología. Y lo rápidos y acertados que sean los gerentes en hacer uso de ella para la toma de las decisiones, que en un mundo tan cambiante y veloz de hoy, pueden significar la vida o la muerte en el mercado.

El hecho de haber encontrado sitios Web de empresas que entre sí tienen relación por ser de idéntico sector, de la misma localidad o clientes de una empresa de servicios multimedia, o realizadas por un determinado equipo de servidores, permite afirmar que la presencia de las grandes empresas manizaleñas en Internet responde a un intento de lograr la ventaja competitiva que supone una diferenciación por posicionamiento tecnológico.

El empleo del correo electrónico como medio para publicitar ciertos dominios, servicios o productos es cada vez más habitual. El e-mail marketing es un fenómeno cada vez más importante en Internet, como vía para contactar clientes de forma más directa. Se podría establecer un paralelo entre lo que es el marketing directo y el e-mail marketing siendo este último una técnica específica para Internet. 
Las 34 grandes empresas de la ciudad de Manizales llevan en promedio dos años conectadas a Internet y aún no han definido estrategias de marketing para llegar a clientes y consumidores que estén interesados en adquirir sus bienes y servicios. Del total de las empresas que son población objetivo, diez grandes empresas llevan más tiempo entre 4 y 5 años con acceso a Internet, han realizado esfuerzos por convertir sus sitios Web interactivos, donde la presencia en Internet está enmarcada en el refuerzo de la imagen corporativa y el ofrecimiento de bienes y servicios.

Un cambio esencial para las grandes empresas consiste en la formulación de estrategias de marketing virtual que complementan el marketing tradicional, de las cuales las más utilizadas son la atención al cliente, servicio post-venta y la investigación de mercados. Otra evidencia de los cambios presentados por la aplicación de Internet en la función de marketing, es que el aumento del número de los clientes no ha sido tan significativo. Sí se han incrementado pero ha sido una pequeña variación. Los entrevistados manifestaron que no hay cultura de compra por Internet, todavía prevalece la inseguridad y la desconfianza por la utilización de este medio. Todavía las grandes empresas de la ciudad de Manizales, no han medido el impacto de su presencia en Internet hacia sus clientes y consumidores. Por esta razón, empresas del sector comercial e industrial sí han encontrado un leve aumento de clientes, mientras que el sector servicios considera lo contrario. Es de resaltar, que el sector servicios en el ámbito mundial es uno de los sectores más prósperos en el uso de las tecnologías Web enfocadas al marketing virtual.

Las grandes empresas del sector industrial se han asesorado con organizaciones de región para reforzar su presencia en Internet. No se han presenciado cambios dentro de la organización relacionadas específicamente con el departamento de marketing. Las grandes empresas que fueron visitadas, conservan una estructura administrativa y organizacional tradicional. En cuanto al sector servicios, el reducido número de empresas que sí han aumentado sus 
clientes por la aplicación de Internet, señalan que han logrado agilizar los trámites en cuanto a pedidos y reservas.

Internet es un instrumento muy flexible, por lo que distintas empresas pueden descubrir potencialidades diferentes y peculiares que se apliquen de manera especialmente adecuada a sus circunstancias comerciales particulares. Pero hay que saber utilizar este instrumento sin olvidar que su función más productiva no debe ser la de sustituir las estrategias de marketing tradicional, sino la de amplificarlas y potenciarlas.

El gran interrogante de las empresas manizaleñas respecto de Internet, es si realmente vale la pena estar allí presentes. En realidad, no existe una única respuesta a este interrogante sino que, como quedó planteado a lo largo del trabajo, cada empresa según su sector tiene su propia justificación. El concepto común en todas ellas es que a través de Internet se tiene un mayor alcance respecto de la audiencia, como así también de brindar servicios adicionales a los clientes, soporte técnico, publicitar, contactar nuevos clientes, entre otros, con menor cantidad de recursos (humanos, temporales y monetarios).

Todo esto, no sólo cobra relevancia a nivel local, ya que se está realizando la inversión a nivel global, y las oportunidades que se le pueden presentar a una empresa sin importar su tamaño son inimaginables; es una forma de conocer y de darse a conocer al mundo entero sin tener que viajar físicamente entre países o continentes.

\section{Referencias}

Bustamante, Claudia Ximena y Fajardo, Isabel Cristina (2001).

Infraestructura de Internet en Colombia: informe final de conectividad nacional. Extraído el 10 de mayo de 2001 desde http://www.crt.gov.co/ proyectos/infra_internet_2000.htm 
Clemente, Peter (1999). El estado de la Net: la nueva frontera. Una mirada reveladora de quién la usa y porqué. 197-209

Doménech, Eduald y Almirón, Nuria (2001). Negocios 3.0. $1^{\text {a }}$ edición, 43

E-marketing: ¿El fin del marketing tradicional? Revista e-jungla. Extraído el 15 de diciembre de 2002 desde http://ejungla.com/covers/covere-marlketing.htm.

Plant, Robert (2001). E-commerce: formulación de una estrategia. 34-55

Stanton, William, Walker, Bruce y Etzel, Michael (2000).

Fundamentos de marketing. $11^{\text {a }}$ edición, 432 